Acta Crystallographica Section E

Structure Reports

Online

ISSN 1600-5368

\section{2-(Phenylsulfanyl)pyridine-3-carboxylic acid}

\section{Muhammad Naeem Khan, ${ }^{\mathrm{a}} \neq$ Misbahul Ain Khan, ${ }^{\mathrm{a}}$ Muhammad Nadeem Arshad, ${ }^{\text {b Islam Ullah Khan }}{ }^{\mathbf{b} *}$ and Salma Rehman}

${ }^{\mathbf{a}}$ Department of Chemistry, Islamia University, Bahawalpur, Pakistan, ${ }^{\mathbf{b}}$ Materials Chemistry laboratory, Department of Chemistry, GC University, Lahore, Pakistan, and 'Applied Chemistry Research Center, PCSIR Laboratories Complex, Lahore 54600, Pakistan

Correspondence e-mail: iukhan.gcu@gmail.com

Received 19 September 2009; accepted 23 September 2009

Key indicators: single-crystal X-ray study; $T=296 \mathrm{~K}$; mean $\sigma(\mathrm{C}-\mathrm{C})=0.006 \AA$; $R$ factor $=0.073 ; w R$ factor $=0.224 ;$ data-to-parameter ratio $=18.5$.

The title compound, $\mathrm{C}_{12} \mathrm{H}_{9} \mathrm{NO}_{2} \mathrm{~S}$, belongs to the nitrogencontaining group of heterocyclic organic compounds and crystallized with two molecules per asymmetric unit. In the crystal, both molecules form inversion dimers linked by pairs of $\mathrm{O}-\mathrm{H}-\mathrm{O}$ hydrogen bonds. Weak symmetry-related $\mathrm{C}-$ $\mathrm{H}-\mathrm{O}$ interactions link the carboxyl dimers along $b$ axis. The dihedral angle between the two aromatic rings in the two molecules are $55.75(14)$ and $58.33(13)^{\circ}$.

\section{Related literature}

For the pharmacological effects of heteroaromatic antitumor compounds: Denny et al. (1982); Fujiwara (1997); Antonini \& Martelli (1992); Cholody et al. (1992). For the title compound as an intermediate for heterocycles, see: Khan et al. $(2008 a, b)$. For the synthesis, see: Mann \& Reid (1952).<smiles>O=C(O)c1cccnc1Sc1ccccc1</smiles>

\section{Experimental}

Crystal data

$\mathrm{C}_{12} \mathrm{H}_{9} \mathrm{NO}_{2} \mathrm{~S}$

$M_{r}=231.26$

Triclinic, $P \overline{1}$

$$
\begin{aligned}
& a=7.2201(4) \AA \\
& b=7.6653(4) \AA \\
& c=19.9537(11) \AA
\end{aligned}
$$

$\ddagger$ Applied Chemistry Research Center, PCSIR Laboratories Complex, Lahore 54600, Pakistan.

$$
\begin{aligned}
& \alpha=97.895(3)^{\circ} \\
& \beta=98.520(3)^{\circ} \\
& \gamma=91.661(3)^{\circ} \\
& V=1080.41(10) \AA^{3} \\
& Z=4
\end{aligned}
$$

Data collection

Bruker Kappa APEXII CCD diffractometer

Absorption correction: multi-scan (SADABS; Bruker, 2005)

$T_{\min }=0.911, T_{\max }=0.983$

\section{Refinement}

$R\left[F^{2}>2 \sigma\left(F^{2}\right)\right]=0.073$

$w R\left(F^{2}\right)=0.224$

$S=1.09$

5397 reflections
Mo $K \alpha$ radiation

$\mu=0.28 \mathrm{~mm}^{-1}$

$T=296 \mathrm{~K}$

\begin{tabular}{|c|c|c|c|c|}
\hline$D-\mathrm{H} \cdots A$ & $D-\mathrm{H}$ & $\mathrm{H} \cdots A$ & $D \cdots A$ & $D-\mathrm{H} \cdots A$ \\
\hline $\mathrm{O} 2-\mathrm{H} 2 \cdots \mathrm{O}^{\mathrm{i}}$ & 0.82 & 1.82 & $2.624(2)$ & $167(1)$ \\
\hline $\mathrm{O} 4-\mathrm{H} 4 \mathrm{o} \cdots \mathrm{O} 1^{\mathrm{i}}$ & 0.82 & 1.83 & $2.642(2)$ & $170(1)$ \\
\hline $\mathrm{C} 3-\mathrm{H} 3 \cdots \mathrm{O} 4^{\mathrm{ii}}$ & 0.93 & 2.50 & $3.264(5)$ & 139 \\
\hline $\mathrm{C} 4-\mathrm{H} 4 \cdots \mathrm{O} 1^{\mathrm{iii}}$ & 0.93 & 2.55 & $3.458(5)$ & 164 \\
\hline $\mathrm{C} 15-\mathrm{H} 15 \cdots \mathrm{O} 2^{\mathrm{ii}}$ & 0.93 & 2.54 & $3.294(5)$ & 138 \\
\hline $\mathrm{C} 16-\mathrm{H} 16 \cdots \mathrm{O} 3^{\mathrm{iii}}$ & 0.93 & 2.58 & $3.467(5)$ & 160 \\
\hline
\end{tabular}

$0.21 \times 0.09 \times 0.06 \mathrm{~mm}$

23200 measured reflections 5397 independent reflections 2766 reflections with $I>2 / \mathrm{s}(I)$ $R_{\text {int }}=0.063$

Table 1

Hydrogen-bond geometry $\left(\AA{ }^{\circ}\right)$.

Symmetry codes: (i) $-x+1,-y,-z$; (ii) $-x+1,-y+1,-z$; (iii) $x, y+1, z$.

Data collection: APEX2 (Bruker, 2007); cell refinement: SAINT (Bruker, 2007); data reduction: $S A I N T$; $\operatorname{program}(\mathrm{s})$ used to solve structure: SHELXS97 (Sheldrick, 2008); program(s) used to refine structure: SHELXL97 (Sheldrick, 2008); molecular graphics: ORTEP-3 for Windows (Farrugia, 1997) and PLATON (Spek, 2009); software used to prepare material for publication: Win $G X$ (Farrugia, 1999) and PLATON.

The authors acknowledge the Higher Education Commission of Pakistan for providing a grant under the project strengthening the Materials Chemistry Laboratory at GC University, Lahore.

Supplementary data and figures for this paper are available from the IUCr electronic archives (Reference: HG2570).

\title{
References
}

Antonini, I. \& Martelli, S. (1992). J. Heterocycl. Chem. 29, 471-473. Bruker (2005). SADABS. Bruker AXS Inc., Madison, Wisconsin, USA.

Bruker (2007). APEX2 and SAINT. Bruker AXS Inc., Madison, Wisconsin, USA.

Cholody, W. M., Martelli, S. \& Konopa, J. (1992). J. Med. Chem. 25, 276-315. Denny, W. A., Cain, B. F., Atwell, G. J., Hansch, C., Panthananickal, A. \& Leo, A. (1982). J. Med. Chem. 25, 276-315.

Farrugia, L. J. (1997). J. Appl. Cryst. 30, 565.

Farrugia, L. J. (1999). J. Appl. Cryst. 32, 837-838.

Fujiwara, H. (1997). Heterocycles, 45, 119-127.

Khan, M. N., Tahir, M. N., Khan, M. A., Khan, I. U. \& Arshad, M. N. (2008a). Acta Cryst. E64, o730.

Khan, M. N., Tahir, M. N., Khan, M. A., Khan, I. U. \& Arshad, M. N. (2008b). Acta Cryst. E64, o1704.

Mann, F. G. \& Reid, J. A. (1952). J. Chem. Soc. pp. 2057-2062.

Sheldrick, G. M. (2008). Acta Cryst. A64, 112-122.

Spek, A. L. (2009). Acta Cryst. D65, 148-155. 


\section{supporting information}

Acta Cryst. (2009). E65, o2662 [https://doi.org/10.1107/S1600536809038586]

\section{2-(Phenylsulfanyl)pyridine-3-carboxylic acid}

\section{Muhammad Naeem Khan, Misbahul Ain Khan, Muhammad Nadeem Arshad, Islam Ullah Khan and Salma Rehman}

\section{S1. Comment}

In continuation of our studies on pyridine-containing heterocyclic compounds, the title compond was synthesized. It is an intermediate for our previously reported crystal structures of 7-nitro-5H-thiochromeno[2,3-b]pyridin-5-one (Khan et al., 2008a) and 5H-thiochromeno[2,3-b]pyridin-5-one (Khan et al., 2008b). Pyridine containing compounds are widely distributed in nature. Heteroaromatic antitumor compounds have been prepared in recent years with the hope of increasing pharmacological effects (Denny et al., 1982), (Fujiwara, 1997), (Antonini \& Martelli, 1992) (Cholody et al., 1992).

The title compound was crystallized with two independent molecules in the asymmetric unit (Fig 1). The dihedral angles between the two aromatic rings in molecule A and molecule B are $55.75(14)^{\circ}$ and $58.33(13)^{\circ}$ respectively. The carboxylic group present in each molecule forms dimers which are linked through weak $\mathrm{C}-\mathrm{H}-\mathrm{O}$ type interaction along the $b$ axis to stabilize the structure Table. $1 \&$ Fig. 2.

\section{S2. Experimental}

A mixture of 2-chloronicotinic acid $(1.57 \mathrm{~g}, 10 \mathrm{mmol})$ and thiophenol $(2 \mathrm{ml})$ was heated under reflux for two hours to produce 2-(Phenylsulfanyl)pyridine-3-carboxylic acid (Mann \& Reid, 1952). Suitable crystals for X-ray diffractions were obtained on cooling the saturated solution of (I) in ethanol.

\section{S3. Refinement}

The $\mathrm{H}$-atoms for aromatic carbons and carboxylic $\mathrm{O}$ atoms were refined geometrically and treated as riding atoms: $\mathrm{C}-\mathrm{H}$ $=0.93 \AA$ with $U_{\text {iso }}(\mathrm{H})=1.2$ and $\mathrm{O}-\mathrm{H}=0.82$ with $U_{\mathrm{iso}}(\mathrm{H})=1.5$. 


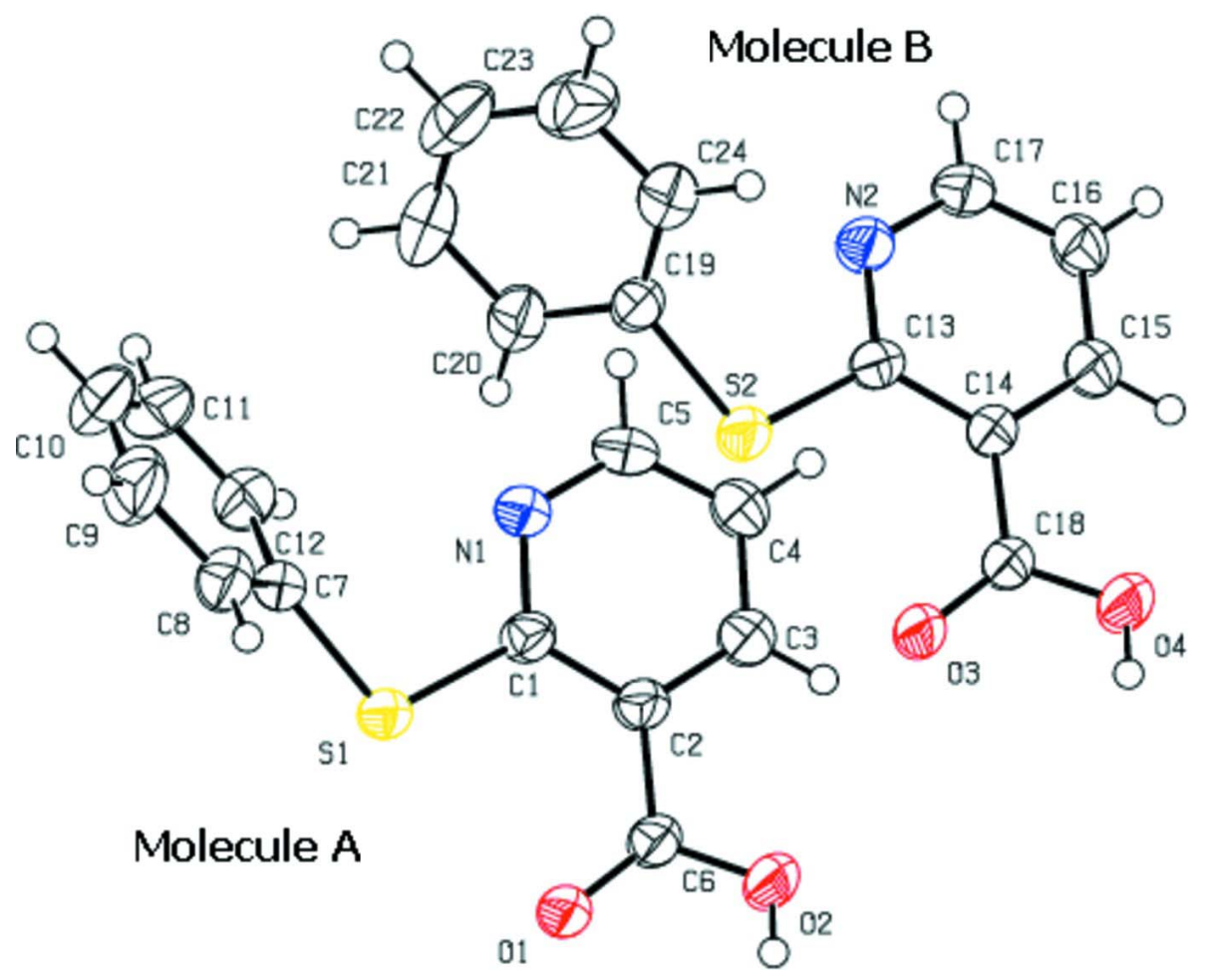

Figure 1

The molecular structure diagram of the title compound showing the atom labels. Thermal ellipsoids are drawn at the $50 \%$ probability level. 


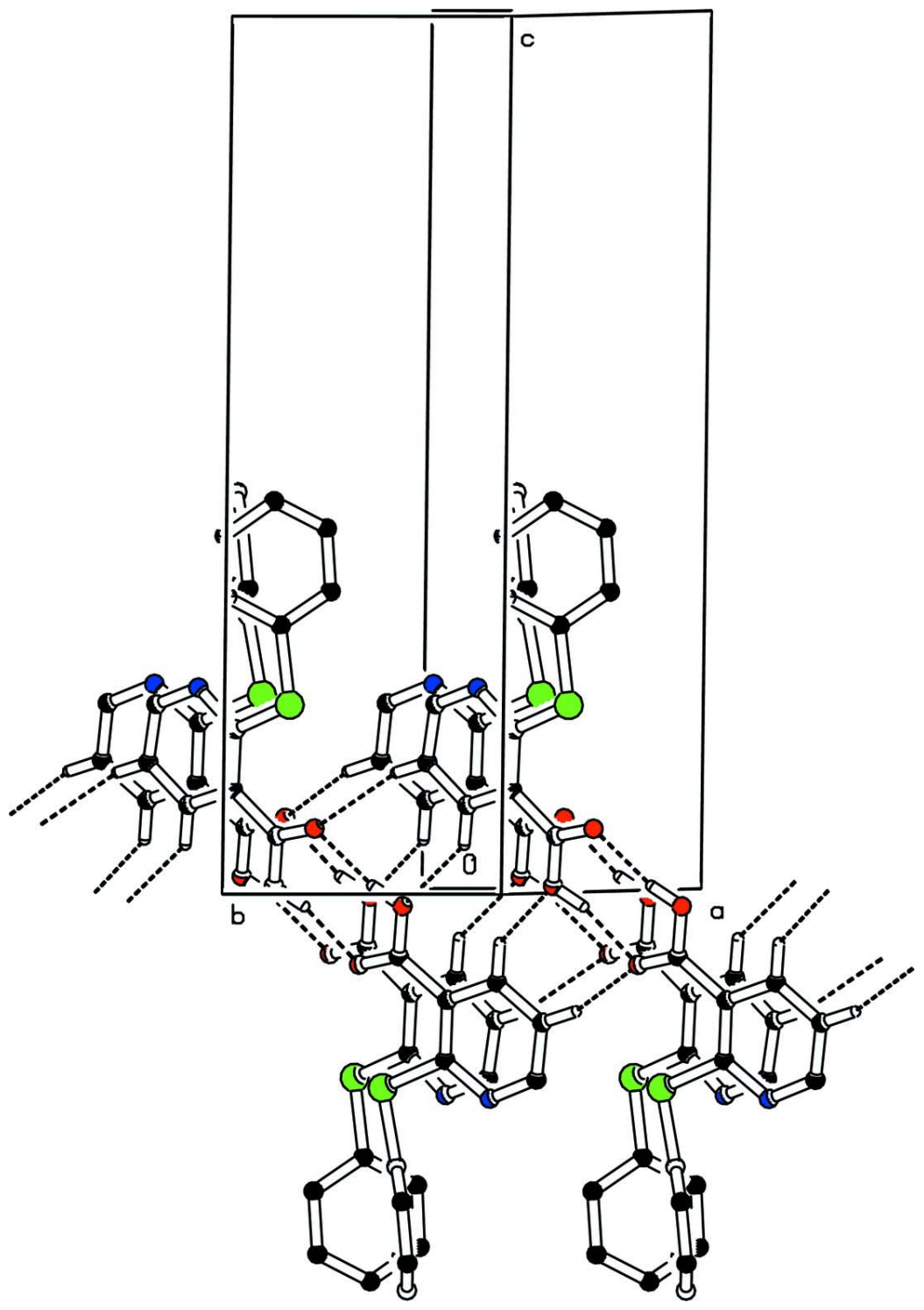

Figure 2

Unit cell packing diagram showing the intermolecular hydrogen bonding using dashed lines. The hydrogen atoms not involved in hydrogen bonding have been omitted.

2-(Phenylsulfanyl)pyridine-3-carboxylic acid

Crystal data

$\mathrm{C}_{12} \mathrm{H}_{9} \mathrm{NO}_{2} \mathrm{~S}$

$M_{r}=231.26$

Triclinic, $P \overline{1}$

Hall symbol: -P 1

$a=7.2201$ (4) $\AA$

$b=7.6653(4) \AA$

$c=19.9537(11) \AA$

$\alpha=97.895(3)^{\circ}$

$\beta=98.520(3)^{\circ}$

$$
\begin{aligned}
& \gamma=91.661(3)^{\circ} \\
& V=1080.41(10) \AA^{3} \\
& Z=4 \\
& F(000)=480 \\
& D_{\mathrm{x}}=1.422 \mathrm{Mg} \mathrm{m}^{-3}
\end{aligned}
$$

Mo $K \alpha$ radiation, $\lambda=0.71073 \AA$

Cell parameters from 3133 reflections

$\theta=2.9-23.8^{\circ}$

$\mu=0.28 \mathrm{~mm}^{-1}$ 
$T=296 \mathrm{~K}$

Needle, white

Data collection

Bruker Kappa APEXII CCD diffractometer

Radiation source: fine-focus sealed tube

Graphite monochromator

$\varphi$ and $\omega$ scans

Absorption correction: multi-scan (SADABS; Bruker, 2005)

$T_{\min }=0.911, T_{\max }=0.983$

Refinement

Refinement on $F^{2}$

Least-squares matrix: full

$R\left[F^{2}>2 \sigma\left(F^{2}\right)\right]=0.073$

$w R\left(F^{2}\right)=0.224$

$S=1.09$

5397 reflections

292 parameters

0 restraints

Primary atom site location: structure-invariant direct methods

Secondary atom site location: difference Fourier map

Special details

Geometry. All e.s.d.'s (except the e.s.d. in the dihedral angle between two 1.s. planes) are estimated using the full covariance matrix. The cell e.s.d.'s are taken into account individually in the estimation of e.s.d.'s in distances, angles and torsion angles; correlations between e.s.d.'s in cell parameters are only used when they are defined by crystal symmetry. An approximate (isotropic) treatment of cell e.s.d.'s is used for estimating e.s.d.'s involving 1.s. planes.

Refinement. Refinement of $F^{2}$ against ALL reflections. The weighted $R$-factor $w R$ and goodness of fit $S$ are based on $F^{2}$, conventional $R$-factors $R$ are based on $F$, with $F$ set to zero for negative $F^{2}$. The threshold expression of $F^{2}>\sigma\left(F^{2}\right)$ is used only for calculating $R$-factors(gt) etc. and is not relevant to the choice of reflections for refinement. $R$-factors based on $F^{2}$ are statistically about twice as large as those based on $F$, and $R$ - factors based on ALL data will be even larger.

Fractional atomic coordinates and isotropic or equivalent isotropic displacement parameters $\left(\AA^{2}\right)$

\begin{tabular}{lllll}
\hline & $x$ & $y$ & $z$ & $U_{\text {iso }} * / U_{\text {eq }}$ \\
\hline S1 & $0.15013(14)$ & $-0.12613(12)$ & $0.21464(5)$ & $0.0407(3)$ \\
S2 & $0.70162(15)$ & $0.36894(12)$ & $0.22267(5)$ & $0.0447(3)$ \\
O1 & $0.1649(4)$ & $-0.2094(3)$ & $0.07364(13)$ & $0.0508(7)$ \\
O2 & $0.2377(6)$ & $-0.0151(4)$ & $0.00790(14)$ & $0.0709(10)$ \\
H2 & 0.2653 & -0.1043 & -0.0155 & $0.106^{*}$ \\
O3 & $0.7077(5)$ & $0.2844(3)$ & $0.08218(13)$ & $0.0568(8)$ \\
O4 & $0.7667(5)$ & $0.4795(4)$ & $0.01516(15)$ & $0.0689(10)$ \\
H4O & 0.7958 & 0.3911 & -0.0083 & $0.103^{*}$ \\
N1 & $0.1813(5)$ & $0.2239(4)$ & $0.23401(15)$ & $0.0422(8)$ \\
N2 & $0.6430(4)$ & $0.7119(4)$ & $0.23511(15)$ & $0.0414(8)$ \\
C1 & $0.1727(5)$ & $0.0818(4)$ & $0.18659(17)$ & $0.0341(8)$ \\
C2 & $0.1832(5)$ & $0.0971(5)$ & $0.11768(18)$ & $0.0382(8)$
\end{tabular}




$\begin{array}{lllll}\text { C3 } & 0.1939(6) & 0.2639(5) & 0.1002(2) & 0.0496(10) \\ \text { H3 } & 0.2005 & 0.2783 & 0.0551 & 0.059^{*} \\ \text { C4 } & 0.1951(6) & 0.4099(5) & 0.1488(2) & 0.0521(11) \\ \text { H4 } & 0.1978 & 0.5234 & 0.1372 & 0.062^{*} \\ \text { C5 } & 0.1921(6) & 0.3809(5) & 0.2150(2) & 0.0485(10) \\ \text { H5 } & 0.1981 & 0.4787 & 0.2486 & 0.058^{*} \\ \text { C6 } & 0.1937(6) & -0.0559(5) & 0.06520(18) & 0.0413(9) \\ \text { C7 } & 0.1766(5) & -0.0719(5) & 0.30494(18) & 0.0380(8) \\ \text { C8 } & 0.3176(6) & -0.1484(5) & 0.34292(19) & 0.0490(10) \\ \text { H8 } & 0.4002 & -0.2190 & 0.3214 & 0.059^{*} \\ \text { C9 } & 0.3357(7) & -0.1189(7) & 0.4143(2) & 0.0656(13) \\ \text { H9 } & 0.4306 & -0.1709 & 0.4403 & 0.079^{*} \\ \text { C10 } & 0.2159(8) & -0.0149(7) & 0.4461(2) & 0.0738(15) \\ \text { H10 } & 0.2290 & 0.0048 & 0.4936 & 0.089^{*} \\ \text { C11 } & 0.0762(7) & 0.0605(6) & 0.4077(2) & 0.0665(14) \\ \text { H11 } & -0.0053 & 0.1320 & 0.4295 & 0.080^{*} \\ \text { C12 } & 0.0542(6) & 0.0326(5) & 0.3379(2) & 0.0509(10) \\ \text { H12 } & -0.0426 & 0.0836 & 0.3125 & 0.061^{*} \\ \text { C13 } & 0.6743(5) & 0.5728(5) & 0.19104(18) & 0.0357(8) \\ \text { C14 } & 0.6895(5) & 0.5891(5) & 0.12249(18) & 0.0373(8) \\ \text { C15 } & 0.6724(6) & 0.7539(5) & 0.1026(2) & 0.0457(10) \\ \text { H15 } & 0.6818 & 0.7686 & 0.0577 & 0.055^{*} \\ \text { C16 } & 0.6416(6) & 0.8959(5) & 0.1483(2) & 0.0463(10) \\ \text { H16 } & 0.6317 & 1.0081 & 0.1358 & 0.056^{*} \\ \text { C17 } & 0.6261(5) & 0.8659(5) & 0.2134(2) & 0.0442(9) \\ \text { H17 } & 0.6017 & 0.9612 & 0.2444 & 0.053^{*} \\ \text { C18 } & 0.7230(6) & 0.4371(5) & 0.07237(18) & 0.0416(9) \\ \text { C19 } & 0.7107(5) & 0.4283(5) & 0.31237(18) & 0.0380(8) \\ \text { C20 } & 0.5879(6) & 0.3405(6) & 0.3442(2) & 0.0492(10) \\ \text { H20 } & 0.4968 & 0.2604 & 0.3184 & 0.059^{*} \\ \text { C21 } & 0.5999(7) & 0.3716(7) & 0.4149(2) & 0.0658(13) \\ \text { H21 } & 0.5161 & 0.3128 & 0.4363 & 0.079^{*} \\ \text { C22 } & 0.7329(8) & 0.4871(7) & 0.4529(2) & 0.0717(14) \\ \text { H22 } & 0.7415 & 0.5066 & 0.5004 & 0.086^{*} \\ \text { C23 } & 0.8545(7) & 0.5748(6) & 0.4213(2) & 0.0683(14) \\ \text { H23 } & 0.9445 & 0.6552 & 0.4476 & 0.082^{*} \\ \text { C24 } & 0.8463(6) & 0.5467(6) & 0.3516(2) & 0.0528(11) \\ \text { H24 } & 0.9308 & 0.6064 & 0.3308 & 0.063^{*} \\ & & & & \end{array}$

Atomic displacement parameters $\left(\AA^{2}\right)$

\begin{tabular}{lllllll}
\hline & $U^{11}$ & $U^{22}$ & $U^{33}$ & $U^{12}$ & $U^{13}$ & $U^{23}$ \\
\hline S1 & $0.0630(7)$ & $0.0289(5)$ & $0.0303(5)$ & $0.0032(4)$ & $0.0088(4)$ & $0.0027(4)$ \\
S2 & $0.0728(7)$ & $0.0313(5)$ & $0.0326(5)$ & $0.0075(5)$ & $0.0147(5)$ & $0.0060(4)$ \\
O1 & $0.092(2)$ & $0.0293(15)$ & $0.0329(15)$ & $0.0027(13)$ & $0.0166(14)$ & $0.0041(12)$ \\
O2 & $0.148(3)$ & $0.0337(16)$ & $0.0387(17)$ & $0.0088(18)$ & $0.0413(19)$ & $0.0042(13)$ \\
O3 & $0.111(2)$ & $0.0291(15)$ & $0.0366(16)$ & $0.0060(14)$ & $0.0284(15)$ & $0.0063(12)$ \\
O4 & $0.137(3)$ & $0.0358(16)$ & $0.0428(18)$ & $0.0118(18)$ & $0.0420(19)$ & $0.0057(13)$
\end{tabular}




$\begin{array}{lllllll}\mathrm{N} 1 & 0.062(2) & 0.0298(17) & 0.0348(18) & 0.0000(14) & 0.0090(15) & 0.0022(14) \\ \mathrm{N} 2 & 0.056(2) & 0.0325(17) & 0.0378(18) & 0.0111(14) & 0.0137(15) & 0.0047(14) \\ \mathrm{C} 1 & 0.041(2) & 0.0320(19) & 0.0291(19) & 0.0071(15) & 0.0034(15) & 0.0037(15) \\ \mathrm{C} 2 & 0.054(2) & 0.0300(19) & 0.0309(19) & 0.0066(16) & 0.0075(16) & 0.0034(15) \\ \mathrm{C} 3 & 0.078(3) & 0.034(2) & 0.040(2) & 0.0069(19) & 0.016(2) & 0.0065(18) \\ \mathrm{C} 4 & 0.084(3) & 0.028(2) & 0.048(3) & 0.0085(19) & 0.016(2) & 0.0099(18) \\ \mathrm{C} 5 & 0.068(3) & 0.029(2) & 0.046(2) & 0.0008(18) & 0.013(2) & -0.0059(18) \\ \mathrm{C} 6 & 0.065(3) & 0.032(2) & 0.029(2) & 0.0089(17) & 0.0106(17) & 0.0056(16) \\ \mathrm{C} 7 & 0.053(2) & 0.0293(19) & 0.031(2) & -0.0026(16) & 0.0100(17) & 0.0019(15) \\ \text { C8 } & 0.063(3) & 0.048(2) & 0.038(2) & 0.005(2) & 0.0113(19) & 0.0104(19) \\ \text { C9 } & 0.077(3) & 0.080(3) & 0.041(3) & -0.003(3) & 0.003(2) & 0.021(2) \\ \text { C10 } & 0.101(4) & 0.087(4) & 0.032(2) & -0.020(3) & 0.020(3) & 0.000(3) \\ \text { C11 } & 0.087(4) & 0.060(3) & 0.054(3) & -0.001(3) & 0.035(3) & -0.010(2) \\ \text { C12 } & 0.063(3) & 0.048(2) & 0.045(2) & 0.004(2) & 0.020(2) & 0.0043(19) \\ \text { C13 } & 0.044(2) & 0.0323(19) & 0.0295(19) & 0.0029(15) & 0.0065(15) & 0.0008(15) \\ \text { C14 } & 0.052(2) & 0.0310(19) & 0.0305(19) & 0.0016(16) & 0.0122(16) & 0.0042(15) \\ \text { C15 } & 0.067(3) & 0.032(2) & 0.041(2) & 0.0050(18) & 0.0151(19) & 0.0080(17) \\ \text { C16 } & 0.064(3) & 0.032(2) & 0.048(2) & 0.0099(18) & 0.016(2) & 0.0126(18) \\ \text { C17 } & 0.060(2) & 0.030(2) & 0.043(2) & 0.0088(17) & 0.0143(18) & -0.0001(17) \\ \text { C18 } & 0.063(3) & 0.032(2) & 0.032(2) & 0.0039(17) & 0.0154(18) & 0.0047(17) \\ \text { C19 } & 0.052(2) & 0.033(2) & 0.0301(19) & 0.0079(16) & 0.0102(16) & 0.0056(16) \\ \text { C20 } & 0.056(3) & 0.053(3) & 0.039(2) & -0.001(2) & 0.0074(19) & 0.0115(19) \\ \text { C21 } & 0.074(3) & 0.087(4) & 0.044(3) & 0.006(3) & 0.023(2) & 0.024(3) \\ \text { C22 } & 0.093(4) & 0.090(4) & 0.033(2) & 0.012(3) & 0.014(3) & 0.005(3) \\ \text { C23 } & 0.079(3) & 0.072(3) & 0.046(3) & 0.004(3) & -0.008(2) & 0.001(2) \\ \text { C24 } & 0.064(3) & 0.053(3) & 0.042(2) & -0.004(2) & 0.009(2) & 0.009(2) \\ & & & & & & \end{array}$

Geometric parameters $\left(\AA,{ }^{o}\right)$

\begin{tabular}{llll}
\hline $\mathrm{S} 1-\mathrm{C} 1$ & $1.771(4)$ & $\mathrm{C} 9-\mathrm{C} 10$ & $1.360(7)$ \\
$\mathrm{S} 1-\mathrm{C} 7$ & $1.773(4)$ & $\mathrm{C} 9-\mathrm{H} 9$ & 0.9300 \\
$\mathrm{~S} 2-\mathrm{C} 13$ & $1.769(4)$ & $\mathrm{C} 10-\mathrm{C} 11$ & $1.365(7)$ \\
$\mathrm{S} 2-\mathrm{C} 19$ & $1.776(4)$ & $\mathrm{C} 10-\mathrm{H} 10$ & 0.9300 \\
$\mathrm{O} 1-\mathrm{C} 6$ & $1.228(4)$ & $\mathrm{C} 11-\mathrm{C} 12$ & $1.365(6)$ \\
$\mathrm{O} 2-\mathrm{C} 6$ & $1.306(4)$ & $\mathrm{C} 11-\mathrm{H} 11$ & 0.9300 \\
$\mathrm{O} 2-\mathrm{H} 2$ & 0.8200 & $\mathrm{C} 12-\mathrm{H} 12$ & 0.9300 \\
$\mathrm{O} 3-\mathrm{C} 18$ & $1.218(4)$ & $\mathrm{C} 13-\mathrm{C} 14$ & $1.411(5)$ \\
$\mathrm{O} 4-\mathrm{C} 18$ & $1.308(4)$ & $\mathrm{C} 14-\mathrm{C} 15$ & $1.379(5)$ \\
$\mathrm{O} 4-\mathrm{H} 4 \mathrm{O}$ & 0.8200 & $\mathrm{C} 14-\mathrm{C} 18$ & $1.478(5)$ \\
$\mathrm{N} 1-\mathrm{C} 5$ & $1.315(5)$ & $\mathrm{C} 15-\mathrm{C} 16$ & $1.367(5)$ \\
$\mathrm{N} 1-\mathrm{C} 1$ & $1.334(4)$ & $\mathrm{C} 15-\mathrm{H} 15$ & 0.9300 \\
$\mathrm{~N} 2-\mathrm{C} 17$ & $1.315(5)$ & $\mathrm{C} 16-\mathrm{C} 17$ & $1.369(5)$ \\
$\mathrm{N} 2-\mathrm{C} 13$ & $1.333(4)$ & $\mathrm{C} 16-\mathrm{H} 16$ & 0.9300 \\
$\mathrm{C} 1-\mathrm{C} 2$ & $1.409(5)$ & $\mathrm{C} 17-\mathrm{H} 17$ & 0.9300 \\
$\mathrm{C} 2-\mathrm{C} 3$ & $1.374(5)$ & $\mathrm{C} 19-\mathrm{C} 20$ & $1.376(5)$ \\
$\mathrm{C} 2-\mathrm{C} 6$ & $1.472(5)$ & $\mathrm{C} 19-\mathrm{C} 24$ & $1.386(5)$ \\
$\mathrm{C} 3-\mathrm{C} 4$ & $1.374(5)$ & $\mathrm{C} 20-\mathrm{C} 21$ & $1.386(5)$ \\
$\mathrm{C} 3-\mathrm{H} 3$ & 0.9300 & $\mathrm{C} 20-\mathrm{H} 20$ & 0.9300
\end{tabular}




\begin{tabular}{|c|c|c|c|}
\hline $\mathrm{C} 4-\mathrm{C} 5$ & $1.372(5)$ & $\mathrm{C} 21-\mathrm{C} 22$ & $1.354(7)$ \\
\hline $\mathrm{C} 4-\mathrm{H} 4$ & 0.9300 & $\mathrm{C} 21-\mathrm{H} 21$ & 0.9300 \\
\hline $\mathrm{C} 5-\mathrm{H} 5$ & 0.9300 & $\mathrm{C} 22-\mathrm{C} 23$ & $1.366(7)$ \\
\hline $\mathrm{C} 7-\mathrm{C} 8$ & $1.372(5)$ & $\mathrm{C} 22-\mathrm{H} 22$ & 0.9300 \\
\hline $\mathrm{C} 7-\mathrm{C} 12$ & $1.387(5)$ & $\mathrm{C} 23-\mathrm{C} 24$ & $1.371(6)$ \\
\hline $\mathrm{C} 8-\mathrm{C} 9$ & $1.397(5)$ & $\mathrm{C} 23-\mathrm{H} 23$ & 0.9300 \\
\hline $\mathrm{C} 8-\mathrm{H} 8$ & 0.9300 & $\mathrm{C} 24-\mathrm{H} 24$ & 0.9300 \\
\hline $\mathrm{C} 1-\mathrm{S} 1-\mathrm{C} 7$ & $103.18(16)$ & $\mathrm{C} 11-\mathrm{C} 12-\mathrm{C} 7$ & $119.9(4)$ \\
\hline $\mathrm{C} 13-\mathrm{S} 2-\mathrm{C} 19$ & $103.20(16)$ & $\mathrm{C} 11-\mathrm{C} 12-\mathrm{H} 12$ & 120.1 \\
\hline $\mathrm{C} 6-\mathrm{O} 2-\mathrm{H} 2$ & 109.5 & $\mathrm{C} 7-\mathrm{C} 12-\mathrm{H} 12$ & 120.1 \\
\hline $\mathrm{C} 18-\mathrm{O} 4-\mathrm{H} 4 \mathrm{O}$ & 109.5 & $\mathrm{~N} 2-\mathrm{C} 13-\mathrm{C} 14$ & $121.1(3)$ \\
\hline $\mathrm{C} 5-\mathrm{N} 1-\mathrm{C} 1$ & $118.8(3)$ & $\mathrm{N} 2-\mathrm{C} 13-\mathrm{S} 2$ & $117.3(3)$ \\
\hline $\mathrm{C} 17-\mathrm{N} 2-\mathrm{C} 13$ & $118.6(3)$ & $\mathrm{C} 14-\mathrm{C} 13-\mathrm{S} 2$ & $121.6(3)$ \\
\hline $\mathrm{N} 1-\mathrm{C} 1-\mathrm{C} 2$ & $121.4(3)$ & $\mathrm{C} 15-\mathrm{C} 14-\mathrm{C} 13$ & $117.9(3)$ \\
\hline $\mathrm{N} 1-\mathrm{C} 1-\mathrm{S} 1$ & $116.8(3)$ & $\mathrm{C} 15-\mathrm{C} 14-\mathrm{C} 18$ & $119.8(3)$ \\
\hline $\mathrm{C} 2-\mathrm{C} 1-\mathrm{S} 1$ & $121.8(3)$ & $\mathrm{C} 13-\mathrm{C} 14-\mathrm{C} 18$ & $122.3(3)$ \\
\hline $\mathrm{C} 3-\mathrm{C} 2-\mathrm{C} 1$ & $117.6(3)$ & $\mathrm{C} 16-\mathrm{C} 15-\mathrm{C} 14$ & $120.5(4)$ \\
\hline $\mathrm{C} 3-\mathrm{C} 2-\mathrm{C} 6$ & $119.1(3)$ & $\mathrm{C} 16-\mathrm{C} 15-\mathrm{H} 15$ & 119.8 \\
\hline $\mathrm{C} 1-\mathrm{C} 2-\mathrm{C} 6$ & $123.1(3)$ & $\mathrm{C} 14-\mathrm{C} 15-\mathrm{H} 15$ & 119.8 \\
\hline $\mathrm{C} 2-\mathrm{C} 3-\mathrm{C} 4$ & $120.7(4)$ & $\mathrm{C} 15-\mathrm{C} 16-\mathrm{C} 17$ & $117.2(3)$ \\
\hline $\mathrm{C} 2-\mathrm{C} 3-\mathrm{H} 3$ & 119.6 & $\mathrm{C} 15-\mathrm{C} 16-\mathrm{H} 16$ & 121.4 \\
\hline $\mathrm{C} 4-\mathrm{C} 3-\mathrm{H} 3$ & 119.6 & $\mathrm{C} 17-\mathrm{C} 16-\mathrm{H} 16$ & 121.4 \\
\hline $\mathrm{C} 5-\mathrm{C} 4-\mathrm{C} 3$ & $117.1(4)$ & $\mathrm{N} 2-\mathrm{C} 17-\mathrm{C} 16$ & $124.7(3)$ \\
\hline $\mathrm{C} 5-\mathrm{C} 4-\mathrm{H} 4$ & 121.5 & $\mathrm{~N} 2-\mathrm{C} 17-\mathrm{H} 17$ & 117.6 \\
\hline $\mathrm{C} 3-\mathrm{C} 4-\mathrm{H} 4$ & 121.5 & $\mathrm{C} 16-\mathrm{C} 17-\mathrm{H} 17$ & 117.6 \\
\hline $\mathrm{N} 1-\mathrm{C} 5-\mathrm{C} 4$ & $124.3(3)$ & $\mathrm{O} 3-\mathrm{C} 18-\mathrm{O} 4$ & $122.1(3)$ \\
\hline $\mathrm{N} 1-\mathrm{C} 5-\mathrm{H} 5$ & 117.8 & $\mathrm{O} 3-\mathrm{C} 18-\mathrm{C} 14$ & $123.5(3)$ \\
\hline $\mathrm{C} 4-\mathrm{C} 5-\mathrm{H} 5$ & 117.8 & $\mathrm{O} 4-\mathrm{C} 18-\mathrm{C} 14$ & $114.3(3)$ \\
\hline $\mathrm{O} 1-\mathrm{C} 6-\mathrm{O} 2$ & $121.9(3)$ & $\mathrm{C} 20-\mathrm{C} 19-\mathrm{C} 24$ & $119.4(4)$ \\
\hline $\mathrm{O} 1-\mathrm{C} 6-\mathrm{C} 2$ & $123.8(3)$ & $\mathrm{C} 20-\mathrm{C} 19-\mathrm{S} 2$ & $117.8(3)$ \\
\hline $\mathrm{O} 2-\mathrm{C} 6-\mathrm{C} 2$ & $114.2(3)$ & $\mathrm{C} 24-\mathrm{C} 19-\mathrm{S} 2$ & $122.5(3)$ \\
\hline $\mathrm{C} 8-\mathrm{C} 7-\mathrm{C} 12$ & $119.6(4)$ & $\mathrm{C} 19-\mathrm{C} 20-\mathrm{C} 21$ & $120.0(4)$ \\
\hline $\mathrm{C} 8-\mathrm{C} 7-\mathrm{S} 1$ & $117.3(3)$ & $\mathrm{C} 19-\mathrm{C} 20-\mathrm{H} 20$ & 120.0 \\
\hline $\mathrm{C} 12-\mathrm{C} 7-\mathrm{S} 1$ & $122.9(3)$ & $\mathrm{C} 21-\mathrm{C} 20-\mathrm{H} 20$ & 120.0 \\
\hline $\mathrm{C} 7-\mathrm{C} 8-\mathrm{C} 9$ & $119.3(4)$ & $\mathrm{C} 22-\mathrm{C} 21-\mathrm{C} 20$ & $120.4(4)$ \\
\hline $\mathrm{C} 7-\mathrm{C} 8-\mathrm{H} 8$ & 120.3 & $\mathrm{C} 22-\mathrm{C} 21-\mathrm{H} 21$ & 119.8 \\
\hline $\mathrm{C} 9-\mathrm{C} 8-\mathrm{H} 8$ & 120.3 & $\mathrm{C} 20-\mathrm{C} 21-\mathrm{H} 21$ & 119.8 \\
\hline $\mathrm{C} 10-\mathrm{C} 9-\mathrm{C} 8$ & $120.6(5)$ & $\mathrm{C} 21-\mathrm{C} 22-\mathrm{C} 23$ & $119.7(4)$ \\
\hline $\mathrm{C} 10-\mathrm{C} 9-\mathrm{H} 9$ & 119.7 & $\mathrm{C} 21-\mathrm{C} 22-\mathrm{H} 22$ & 120.2 \\
\hline $\mathrm{C} 8-\mathrm{C} 9-\mathrm{H} 9$ & 119.7 & $\mathrm{C} 23-\mathrm{C} 22-\mathrm{H} 22$ & 120.2 \\
\hline $\mathrm{C} 9-\mathrm{C} 10-\mathrm{C} 11$ & $119.6(4)$ & $\mathrm{C} 22-\mathrm{C} 23-\mathrm{C} 24$ & $121.3(4)$ \\
\hline $\mathrm{C} 9-\mathrm{C} 10-\mathrm{H} 10$ & 120.2 & $\mathrm{C} 22-\mathrm{C} 23-\mathrm{H} 23$ & 119.3 \\
\hline $\mathrm{C} 11-\mathrm{C} 10-\mathrm{H} 10$ & 120.2 & $\mathrm{C} 24-\mathrm{C} 23-\mathrm{H} 23$ & 119.3 \\
\hline $\mathrm{C} 10-\mathrm{C} 11-\mathrm{C} 12$ & $121.1(4)$ & $\mathrm{C} 23-\mathrm{C} 24-\mathrm{C} 19$ & $119.3(4)$ \\
\hline $\mathrm{C} 10-\mathrm{C} 11-\mathrm{H} 11$ & 119.5 & $\mathrm{C} 23-\mathrm{C} 24-\mathrm{H} 24$ & 120.4 \\
\hline $\mathrm{C} 12-\mathrm{C} 11-\mathrm{H} 11$ & 119.5 & $\mathrm{C} 19-\mathrm{C} 24-\mathrm{H} 24$ & 120.4 \\
\hline
\end{tabular}




$\begin{array}{llll}\mathrm{C} 5-\mathrm{N} 1-\mathrm{C} 1-\mathrm{C} 2 & 2.8(5) & \mathrm{C} 17-\mathrm{N} 2-\mathrm{C} 13-\mathrm{C} 14 & 0.1(5) \\ \mathrm{C} 5-\mathrm{N} 1-\mathrm{C} 1-\mathrm{S} 1 & -177.9(3) & \mathrm{C} 17-\mathrm{N} 2-\mathrm{C} 13-\mathrm{S} 2 & 178.6(3) \\ \mathrm{C} 7-\mathrm{S} 1-\mathrm{C} 1-\mathrm{N} 1 & -7.2(3) & \mathrm{C} 19-\mathrm{S} 2-\mathrm{C} 13-\mathrm{N} 2 & -8.3(3) \\ \mathrm{C} 7-\mathrm{S} 1-\mathrm{C} 1-\mathrm{C} 2 & 172.1(3) & \mathrm{C} 19-\mathrm{S} 2-\mathrm{C} 13-\mathrm{C} 14 & 170.2(3) \\ \mathrm{N} 1-\mathrm{C} 1-\mathrm{C} 2-\mathrm{C} 3 & -2.9(6) & \mathrm{N} 2-\mathrm{C} 13-\mathrm{C} 14-\mathrm{C} 15 & 0.4(6) \\ \mathrm{S} 1-\mathrm{C} 1-\mathrm{C} 2-\mathrm{C} 3 & 177.8(3) & \mathrm{S} 2-\mathrm{C} 13-\mathrm{C} 14-\mathrm{C} 15 & -177.9(3) \\ \mathrm{N} 1-\mathrm{C} 1-\mathrm{C} 2-\mathrm{C} 6 & 173.5(3) & \mathrm{N} 2-\mathrm{C} 13-\mathrm{C} 14-\mathrm{C} 18 & -179.6(3) \\ \mathrm{S} 1-\mathrm{C} 1-\mathrm{C} 2-\mathrm{C} 6 & -5.8(5) & \mathrm{S} 2-\mathrm{C} 13-\mathrm{C} 14-\mathrm{C} 18 & 2.0(5) \\ \mathrm{C} 1-\mathrm{C} 2-\mathrm{C} 3-\mathrm{C} 4 & 0.2(6) & \mathrm{C} 13-\mathrm{C} 14-\mathrm{C} 15-\mathrm{C} 16 & 0.0(6) \\ \mathrm{C} 6-\mathrm{C} 2-\mathrm{C} 3-\mathrm{C} 4 & -176.3(4) & \mathrm{C} 18-\mathrm{C} 14-\mathrm{C} 15-\mathrm{C} 16 & -179.9(4) \\ \mathrm{C} 2-\mathrm{C} 3-\mathrm{C} 4-\mathrm{C} 5 & 2.4(6) & \mathrm{C} 14-\mathrm{C} 15-\mathrm{C} 16-\mathrm{C} 17 & -1.0(6) \\ \mathrm{C} 1-\mathrm{N} 1-\mathrm{C} 5-\mathrm{C} 4 & 0.1(6) & \mathrm{C} 13-\mathrm{N} 2-\mathrm{C} 17-\mathrm{C} 16 & -1.3(6) \\ \mathrm{C} 3-\mathrm{C} 4-\mathrm{C} 5-\mathrm{N} 1 & -2.6(7) & \mathrm{C} 15-\mathrm{C} 16-\mathrm{C} 17-\mathrm{N} 2 & 1.7(6) \\ \mathrm{C} 3-\mathrm{C} 2-\mathrm{C} 6-\mathrm{O} 1 & -171.7(4) & \mathrm{C} 15-\mathrm{C} 14-\mathrm{C} 18-\mathrm{O} 3 & -166.9(4) \\ \mathrm{C} 1-\mathrm{C} 2-\mathrm{C} 6-\mathrm{O} 1 & 12.0(6) & \mathrm{C} 13-\mathrm{C} 14-\mathrm{C} 18-\mathrm{O} 3 & 13.2(6) \\ \mathrm{C} 3-\mathrm{C} 2-\mathrm{C} 6-\mathrm{O} 2 & 8.6(6) & \mathrm{C} 15-\mathrm{C} 14-\mathrm{C} 18-\mathrm{O} 4 & 11.6(6) \\ \mathrm{C} 1-\mathrm{C} 2-\mathrm{C} 6-\mathrm{O} 2 & -167.8(4) & \mathrm{C} 13-\mathrm{C} 14-\mathrm{C} 18-\mathrm{O} 4 & -168.3(4) \\ \mathrm{C} 1-\mathrm{S} 1-\mathrm{C} 7-\mathrm{C} 8 & -122.4(3) & \mathrm{C} 13-\mathrm{S} 2-\mathrm{C} 19-\mathrm{C} 20 & 126.3(3) \\ \mathrm{C} 1-\mathrm{S} 1-\mathrm{C} 7-\mathrm{C} 12 & 62.3(4) & \mathrm{C} 13-\mathrm{S} 2-\mathrm{C} 19-\mathrm{C} 24 & -59.6(4) \\ \mathrm{C} 12-\mathrm{C} 7-\mathrm{C} 8-\mathrm{C} 9 & -0.2(6) & \mathrm{C} 24-\mathrm{C} 19-\mathrm{C} 20-\mathrm{C} 21 & 0.3(6) \\ \mathrm{S} 1-\mathrm{C} 7-\mathrm{C} 8-\mathrm{C} 9 & -175.7(3) & \mathrm{S} 2-\mathrm{C} 19-\mathrm{C} 20-\mathrm{C} 21 & 174.6(3) \\ \mathrm{C} 7-\mathrm{C} 8-\mathrm{C} 9-\mathrm{C} 10 & -0.3(7) & \mathrm{C} 19-\mathrm{C} 20-\mathrm{C} 21-\mathrm{C} 22 & -0.5(7) \\ \mathrm{C} 8-\mathrm{C} 9-\mathrm{C} 10-\mathrm{C} 11 & 0.3(8) & \mathrm{C} 20-\mathrm{C} 21-\mathrm{C} 22-\mathrm{C} 23 & 0.7(8) \\ \mathrm{C} 9-\mathrm{C} 10-\mathrm{C} 11-\mathrm{C} 12 & 0.3(8) & \mathrm{C} 21-\mathrm{C} 22-\mathrm{C} 23-\mathrm{C} 24 & -0.8(8) \\ \mathrm{C} 10-\mathrm{C} 11-\mathrm{C} 12-\mathrm{C} 7 & -0.8(7) & \mathrm{C} 22-\mathrm{C} 23-\mathrm{C} 24-\mathrm{C} 19 & 0.7(7) \\ \mathrm{C} 8-\mathrm{C} 7-\mathrm{C} 12-\mathrm{C} 11 & 0.8(6) & \mathrm{C} 20-\mathrm{C} 19-\mathrm{C} 24-\mathrm{C} 23 & -0.4(6) \\ \mathrm{S} 1-\mathrm{C} 7-\mathrm{C} 12-\mathrm{C} 11 & 176.0(3) & \mathrm{S} 2-\mathrm{C} 19-\mathrm{C} 24-\mathrm{C} 23 & -174.4(3) \\ & & & \end{array}$

Hydrogen-bond geometry $\left(\AA,{ }^{\circ}\right)$

\begin{tabular}{lllll}
\hline$D-\mathrm{H} \cdots A$ & $D-\mathrm{H}$ & $\mathrm{H} \cdots A$ & $D \cdots A$ & $D-\mathrm{H} \cdots A$ \\
\hline $\mathrm{O} 2-\mathrm{H} 2 \cdots \mathrm{O} 3^{\mathrm{i}}$ & 0.82 & 1.82 & $2.624(2)$ & $168(1)$ \\
$\mathrm{O} 4-\mathrm{H} 40 \cdots \mathrm{O} 1^{\mathrm{i}}$ & 0.82 & 1.83 & $2.642(2)$ & $170(1)$ \\
$\mathrm{C} 3-\mathrm{H} 3 \cdots \mathrm{O} 4^{\mathrm{ii}}$ & 0.93 & 2.50 & $3.264(5)$ & 139 \\
$\mathrm{C} 4-\mathrm{H} 4 \cdots{ }^{\mathrm{iii}}$ & 0.93 & 2.55 & $3.458(5)$ & 164 \\
$\mathrm{C} 15-\mathrm{H} 15 \cdots \mathrm{O} 2^{\mathrm{ii}}$ & 0.93 & 2.54 & $3.294(5)$ & 138 \\
$\mathrm{C} 16-\mathrm{H} 16 \cdots 3^{\mathrm{iii}}$ & 0.93 & 2.58 & $3.467(5)$ & 160 \\
\hline
\end{tabular}

Symmetry codes: (i) $-x+1,-y,-z$; (ii) $-x+1,-y+1,-z$; (iii) $x, y+1, z$. 